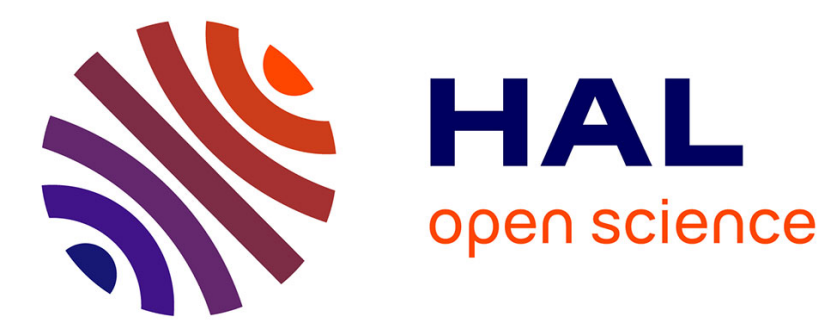

\title{
Spurious phase correction in rapid metabolic imaging
}

Nour El Sabbagh, Carine Chassain, H. Ratiney, Guilhem Pagés, J.-M. Bonny

\section{To cite this version:}

Nour El Sabbagh, Carine Chassain, H. Ratiney, Guilhem Pagés, J.-M. Bonny. Spurious phase correction in rapid metabolic imaging. Journal of Magnetic Resonance, 2021, 332 (107065), pp.107065. 10.1016/j.jmr.2021.107065 . hal-03363454

\section{HAL Id: hal-03363454 https://hal.inrae.fr/hal-03363454}

Submitted on 4 Oct 2021

HAL is a multi-disciplinary open access archive for the deposit and dissemination of scientific research documents, whether they are published or not. The documents may come from teaching and research institutions in France or abroad, or from public or private research centers.
L'archive ouverte pluridisciplinaire HAL, est destinée au dépôt et à la diffusion de documents scientifiques de niveau recherche, publiés ou non, émanant des établissements d'enseignement et de recherche français ou étrangers, des laboratoires publics ou privés. 


\title{
Communication
}

\section{Spurious phase correction in rapid metabolic imaging}

\author{
Nour El Sabbagh ${ }^{a, b, c, *}$, Carine Chassain ${ }^{a, b, c}$, Hélène Ratiney ${ }^{\mathrm{d}}$, Guilhem Pagés ${ }^{\mathrm{a}, \mathrm{b}}$, \\ Jean-Marie Bonny ${ }^{\mathrm{a}, \mathrm{b}}$ \\ a INRAE, UR QuaPA, F-63122 Saint-Gènes-Champanelle, France \\ ${ }^{\mathrm{b}}$ INRAE, AgroResonance Facility, F-63122 Saint-Genès-Champanelle, France \\ ${ }^{\mathrm{c}}$ Université Clermont Auvergne, CHU, CNRS, Clermont Auvergne INP, Institut Pascal, F-63000 Clermont-Ferrand, France \\ d Univ Lyon, INSA-Lyon, Université Claude Bernard Lyon 1, UJM Saint Etienne, CNRS, Inserm, CREATIS UMR5220, U1294, F-69621, LYON, France
}

\section{A R T I C L E I N F O}

\section{Article history:}

Received 9 July 2021

Revised 6 September 2021

Accepted 6 September 2021

Available online 8 September 2021

\section{Keyword:}

MRSI

Multi-shot imaging;

Phase correction

Frequency commutation

Phase-coherent

Phase-continuous

\begin{abstract}
A B S T R A C T
IDEAL-type magnetic resonance spectroscopic imaging (MRSI) sequences require the acquisition of several datasets using optimized sampling in the time domain to reconstruct metabolite maps. Each unitary scan consists of a selective slice (2D) or slab (3D) excitation followed by an evolution time and then the acquisition of the spatially encoded signal. It is critical that the phase variation during the evolution time for each scan is only dependent on chemical shifts. In this paper, we described the apparition of spurious phase due to either the transmit or the receive frequency. The presence of this unwanted phase depends on (i) where the commutation between these two frequencies is performed and (ii) how it is done, as there are two phase commutation modes: continuous and coherent. We present the correction needed in function of the different cases. It appears that some solutions are universal. However, it is critical to know which case is implemented on the MRI scanner, which is not always easy information to have. We illustrated several cases with our preclinical MRI by using the IDEAL spiral method on a ${ }^{13} \mathrm{C}$ phantom.
\end{abstract} (c) 2021 Elsevier Inc. All rights reserved.

\section{Introduction}

Magnetic resonance spectroscopic imaging (MRSI) maps metabolite spatial distribution by exploiting the information contained in the chemical shift (CS). Applications have been used for tumour/lesion identification, treatment and survivorship studies [1,2], tracking changes in metabolic pathways [3-7], fat/water imaging [8-10], and magnetic resonance thermography or quantitative temperature imaging $[11,12]$. In order to record both spatial and spectral information, different methods were developed.

The first method is the conventional phase-encoded MRSI, currently known as chemical shift imaging (CSI), where the phase encoding gradient amplitude is incremented once per repetition time (TR), leading to a complete separation of the spectral and spatial encoding [13]. Data reconstruction is therefore straightforward, using Fourier transforms. This method offers high sensitivity and localisation performance, but with long acquisition times. To overcome this drawback, the time to acquire either spatial or spectral information must be shortened. One of the most popular approaches to significantly decreasing MRSI experimental

\footnotetext{
* Corresponding author at: INRAE, Route de Theix, 63122 Saint-Genès-Champanelle, France.

E-mail address: Nour.El-Sabbagh@inrae.fr (N. El Sabbagh).
}

time is the echo planar spectroscopic imaging (EPSI) acquisition scheme, introduced by Mansfield [14]. It is based on a series of periodically inverted gradients, generating a train of echoes, from which both spatial and spectral information can be extracted within one scan. However, increasing the spatial resolution leads to a decrease in the spectral one and vice-versa. Such limitations prevent having a high resolution in both the spatial and spectral dimensions, especially at high magnetic fields.

In order to make the resolutions independent of one another, one solution is to quickly acquire several images recorded at different echo times (TEs). Utilizing this strategy, the spatial information is directly recorded with single-shot trajectories (e.g., EPI, spiral. . .) $[15,16]$. As for the spectral information, it is extracted from the signal evolution at different TEs using the iterative decomposition of water and fat with echo asymmetry and least-squares estimation (IDEAL) concept $[7,9,17,18]$. Fig. 1 illustrates the principle of the IDEAL method combined with a spiral encoding, to give the 2D IDEAL spiral [15]. Such method is particularly useful for hyperpolarized ${ }^{13} \mathrm{C}$ metabolic mapping [15]. After a selective slice excitation during $t_{1}$, the spin system is allowed to evolve for a time duration of $t_{2}-t_{1}$ followed by a spiral trajectory image recording. The CS information is recorded through the analysis of the phase evolution occurring during the different shots. This phase evolution depends on the frequency difference between the isochromats 


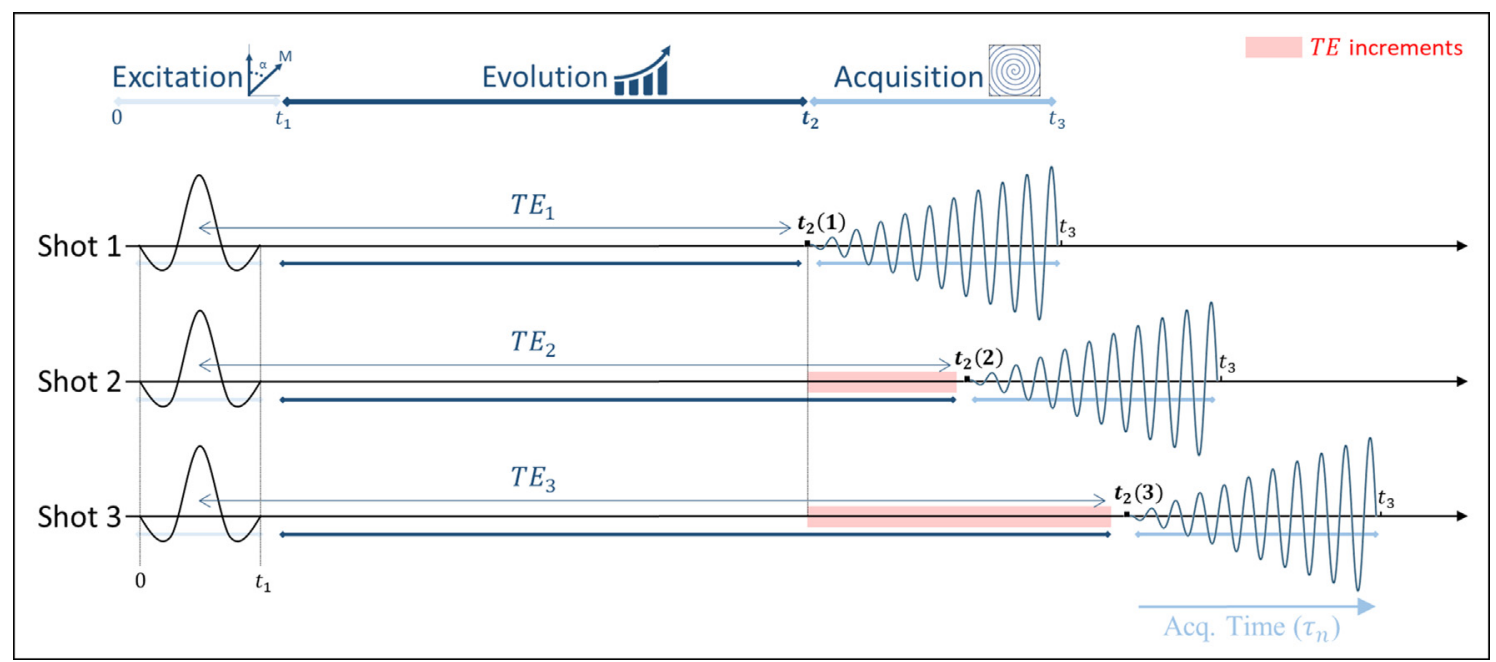

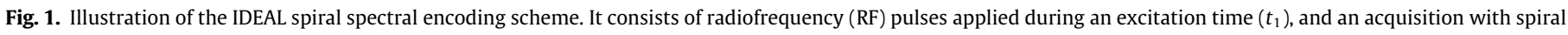
spatial encoding $\left(t_{3}-t_{2}\right)$ applied after an evolution time $\left(t_{2}(m)-t_{1}\right)$. Several shots with different evolution times are used to encode the spectral information.

and the rotating detection frame. To both limit the number of TEs and optimize the TE increments, it is important to have a sparse NMR spectrum as well as prior knowledge on the chemical shift values. In addition, the experiment timing needs to be optimized through the careful choice of the number of signal averages (NSA) $[15,17]$. Once the experiment is recorded, the various data obtained from the different shots are analysed as one to reconstruct one map per metabolite, as explained in [15]. To obtain a correct reconstruction, it is thus mandatory to get a correct phase, i.e., not prone to spurious influence. Rapid 3D encoding is also possible [19], but obtaining a higher spatial resolution in three dimensions is problematic. Several artefacts, such as motion, may introduce unwanted phase terms. To correct for this, several approaches have been developed [14,20-23]. Another source of phase errors that we address can occur in 2D or 3D with slab selection. Indeed, the radiofrequency (RF) excitation is applied with the transmit frequency $\left(f_{T}\right)$ and the acquisition is done in reference to the receive frequency $\left(f_{R}\right)$. A commutation between these two frequencies has to be made during the pulse sequence. However, depending on the mode and the position of the commutation, phase errors may occur. Two modes of commutation are considered and elaborated later: phase-continuous and phase-coherent switching [24].

The aim of this paper is to elucidate the impact of the frequency commutation's mode and position on the metabolite map obtained by 2D IDEAL-type method and to show how to overcome the resulting phase errors. This is, to our knowledge, not described in the literature and yet very critical. To experimentally validate this, the IDEAL spiral was applied to a ${ }^{13} \mathrm{C}$ phantom.

\section{Theory}

The reconstruction of metabolite maps from IDEAL-type sequences requires that the phase variation between shots is only dependent on the CS. All frequencies mentioned in the following are offset values from the reference frequency of the rotating detection frame. In 2D MRI pulse sequences, frequency selective pulses are performed at $f_{T}$, which depends on both the slice position and the slice selection gradient $G_{z}$ intensity. The signal acquisition is performed at $f_{R}$.

To the best of our knowledge, there are two modes to commute from $f_{T} \rightarrow f_{R}$ set on both clinical and preclinical MRI scanners. The first one is the phase-continuous switch, where the rotation of the receiver frame starts from the phase where the rotating transmitter frame ended (i.e., $2 \pi f_{T} t$, for a commutation at a time $t$ ). The second one is the phase-coherent switch. A numerically controlled oscillator (NCO) is used to count the phase of the reference frame rotating at the on-resonance frequency. At the commutation moment $t$, the rotation of the receiver frame starts from the phase accumulated by the NCO up to this instant [24].

Fig. 2 illustrates the different cases of the transmit/receive frequency commutation during pulse sequence timing. We identified three options: a commutation before the signal acquisition, one after the slice selection or a combination of the two. In our case, the signal acquired during the $m^{\text {th }}$ shot and at the $n^{\text {th }} \mathrm{k}$-space sample location $\boldsymbol{k}_{n}$ (at the instant $\tau_{n}$ ) can be formulated as:

$S_{m, n}=\sum_{p, q} e^{i \boldsymbol{k}_{n} \boldsymbol{r}_{p}} e^{i 2 \pi\left[\left(f_{T}+f_{q}\right) t_{1}+\left(f_{\text {evol }}+f_{q}\right)\left(t_{2}(m)-t_{1}\right)+\left(f_{R}+f_{q}\right) \tau_{n}\right]} X_{q}\left(\boldsymbol{r}_{p}\right)$,

where $f_{q}$ and $X_{q}(\boldsymbol{r})$ represent the $q^{\text {th }}$ metabolite frequency and its spatial distribution in the image space, respectively; $\left(t_{2}(m)-t_{1}\right)$ the evolution time of the $m^{\text {th }}$ shot and $f_{\text {evol }}$ being the frequency of the rotating frame during this time.

In the following sections, we present the three sequence configurations and only phases influenced by the pulse sequence are addressed and analysed, and not those owed to the CS. Therefore, to successfully maintain the unique-dependence of phase to CS during evolution time, the phase variation between shots at $t_{2}(m)$ (i.e., $\left.\Delta \phi\left(t_{2}\right)\right)$ should be nil. We considered that the phase accumulated up to $t_{1}$ is identical for all shots. In other words, a phase-coherent return $f_{R} \rightarrow f_{T}$ is done before the following excitation pulse, resulting in the same starting phase at the beginning of each shot.

Acq-Switch: Switch from $f_{T}$ to $f_{R}$ at $t_{2}$

For this first case, the phase-continuous frequency commutation from $f_{T}$ to $f_{R}$ is done once at $t_{2}(m)$, i.e., before the acquisition, as presented in Fig. 2 and leading to $f_{\text {evol }}=f_{T}$. Here, the phase accumulated at the beginning of the acquisition for the $m^{\text {th }}$ shot is

$\phi_{m}\left(t_{2}\right)=2 \pi f_{T} t_{2}(m)$.

The phase increment between shots can be expressed as

$\Delta \phi_{t_{2}}=\phi_{m+1}\left(t_{2}\right)-\phi_{m}\left(t_{2}\right)=2 \pi f_{T}\left(t_{2}(m+1)-t_{2}(m)\right)$.

One can readily see that the phase increment is dependent on $f_{T}$, i.e., the slice position. As soon as the slice is not centered, a phase accumulation due to $f_{T}$ is added to the CS-dependent phase. 


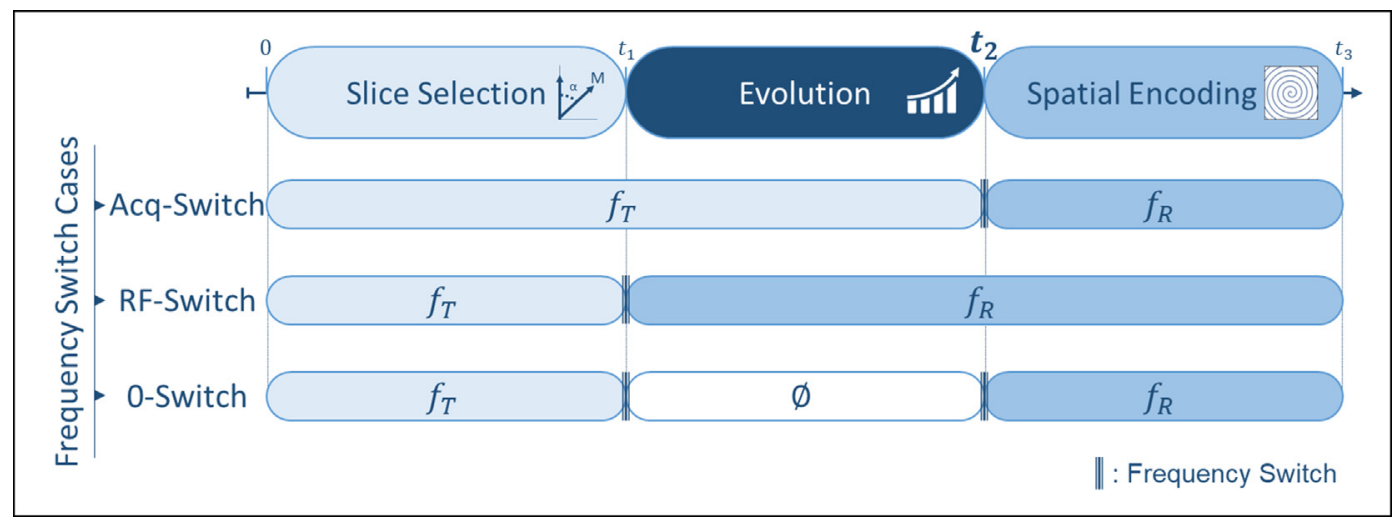

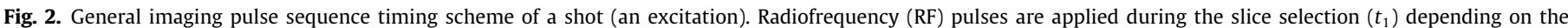

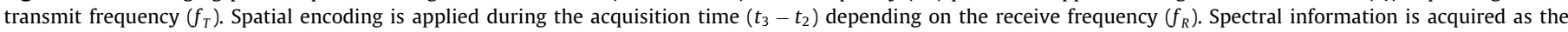

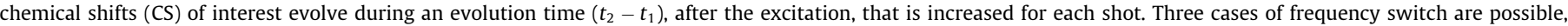

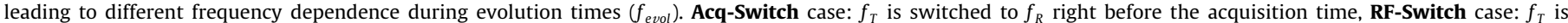

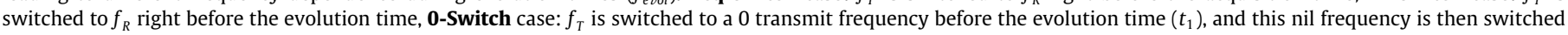
back to $f_{R}$ at the end of it $\left(t_{2}\right)$.

In practice, this phase shift can be large $\left(\Delta \phi_{t_{2}} \gg 2 \pi\right)$ compared to the CS-related one and can thus appear to be random due to phase wrapping. To give an order of magnitude, let us consider a pulse bandwidth (BW) of $1 \mathrm{kHz}$ selecting a slice thickness of $1 \mathrm{~mm}$ and shifted by $5 \mathrm{~mm}$ from the magnet's centre. During a TE of $1 \mathrm{~ms}$, the phase accumulated in this slice is $10 \pi$. Incrementing the TE by $0.5 \mathrm{~ms}$ adds a spurious phase of $5 \pi$. This shot-dependent spurious phase must be removed for each data point $\left(k_{n}\right)$ before metabolite map reconstruction, i.e.,

$S_{m, n}^{\prime}=S_{m, n} e^{-i 2 \pi f_{T}\left(t_{2}(m)-t_{2}(1)\right)}$,

where $S^{\prime}$ and $S$ are the corrected and the raw acquired signal, respectively. The signal has to be corrected with respect to the first shot.

When the frequency commutation from $f_{T}$ to $f_{R}$ at $t_{2}$ is done in the phase-coherent mode, the rotation of the receiver frame starts from the phase accumulated by the reference rotating frame at this time, achieved by using an NCO. The phase variation due to the excitation is then cancelled and therefore, no spurious phase is accumulated.

RF-Switch: switch from $f_{T}$ to $f_{R}$ at $t_{1}$

In the second case, the phase-continuous frequency commutation from $f_{T}$ to $f_{R}$ is done right after the excitation, at $t_{1}$. The phase variation between consecutive shots depends here on the receive frequency, i.e.,

$\Delta \phi_{t_{2}}=\phi_{m+1}\left(t_{2}\right)-\phi_{m}\left(t_{2}\right)=2 \pi f_{R}\left(t_{2}(m+1)-t_{2}(m)\right)$.

Most of the single-shot trajectories require keeping $f_{R}$ nil to not shift the k-space trajectory. No spurious phase shifts are accumulated between shots $\left(\Delta \phi_{t_{2}}=0\right)$, and therefore, no correction is needed. In the case of $f_{R} \neq 0$, e.g., for introducing an off-centre shift as in spin-warp encoding [25], the resulting spurious phase should be corrected in the acquired signal as in Eq. (4) but by substituting $f_{T}$ by $f_{R}$, i.e.,

$S_{m, n}^{\prime}=S_{m, n} e^{-i 2 \pi f_{R}\left(t_{2}(m)-t_{2}(1)\right)}$

Since the commutation is done before echo time increments at $t_{1}$, the spurious phase problem persists for $f_{R} \neq 0$ in the phasecoherent mode, and the correction presented in Eq. (6) remains crucial.

0-Switch: double switch, from $f_{T}$ to 0 at $t_{1}$ and from 0 to $f_{R}$ at $t_{2}$

In the last case, a double switch is done during the pulse sequence. A first switch from the transmit frequency $f_{T}$ to a 0 one is done right before the evolution time at $t_{1}$, and then switched again to the receive frequency $f_{R}$ at $t_{2}$. In this case, for both phasecontinuous and phase-coherent modes, no additional phase is accumulated at $t_{2}$ even if the slice and/or the readout position are shifted.

Phase correction requirements for the three commutation cases (Acq-, RF- and 0-Switch) and in the two switch modes (phasecontinuous and -coherent modes), are summarized in Table 1.

\section{Method}

All the experiments were performed on a BRUKER 117/16 $(11.7 \mathrm{~T})$ horizontal bore magnet equipped with a BGA-9 gradient coil (BRUKER BioSpin MRI GmbH). The magnet reference resonance frequencies were 500.34 and $125.83 \mathrm{MHz}$ for ${ }^{1} \mathrm{H}$ and ${ }^{13} \mathrm{C}$, respectively.

The phantom was composed of four syringes filled with either $\left[1-{ }^{13} \mathrm{C}\right.$ Pyruvic acid $(16.4 \mathrm{M}, \sim 0 \mathrm{~Hz}),\left[1-{ }^{13} \mathrm{C}\right]$ Lactate $(4 \mathrm{M}$, $\sim 985 \mathrm{~Hz}),\left[1-{ }^{13} \mathrm{C}\right]$ Alanine $(1.85 \mathrm{M}, \sim 135 \mathrm{~Hz})$ or $\left[1-{ }^{13} \mathrm{C}\right]$ Urea $(16.4 \mathrm{M}, \sim-1500 \mathrm{~Hz})$ fixed in $10 \%$ gelatine. $\mathrm{A}{ }^{1} \mathrm{H} /{ }^{13} \mathrm{C}$ volume coil (72 mm internal diameter; RAPID Biomedical GmbH, Rimpar, Germany) was used to excite both nuclei and receive the ${ }^{1} \mathrm{H}$ signal. ${ }^{13} \mathrm{C}$

Table 1

Phase correction requirements during IDEAL-type sequences for Acq-, RF- and 0-Switch cases and in the phase-continuous and phase-coherent $f_{T} \rightarrow f_{R}$ switch modes.

\begin{tabular}{|c|c|c|c|}
\hline & & \multicolumn{2}{|c|}{ Switch Modes } \\
\hline & & Phase-Continuous & Phase-Coherent \\
\hline Switch Cases & $\begin{array}{l}\text { Acq-Switch } \\
\text { RF-Switch } \\
\text { 0-Switch }\end{array}$ & $\begin{array}{r}\text { Correction if } f_{T} \neq 0 \text { following Eq. (4) } \\
\text { Correction } \mathrm{i}\end{array}$ & No Correction needed \\
\hline
\end{tabular}


signal reception used a single-loop rat brain surface coil (RAPID Biomedical GmbH, Rimpar, Germany). ${ }^{1} \mathrm{H}$ images were recorded with a fast low angle shot method (FLASH) to validate the phantom position inside the magnet. For ${ }^{13} \mathrm{C}$, a $15 \mathrm{~mm}$ thick slice was set to make sure a sufficient signal to noise ratio (SNR) was reached in a reasonable time. The field of view (FOV) was $50 \times 50 \mathrm{~mm}^{2}$ with an image matrix of $64 \times 64$ and a reception BW of $400 \mathrm{kHz}$. Each one of the RF excitation pulses used was a Shinnar-Le Roux (SLR) set to $90^{\circ}$ with a duration of $2.1 \mathrm{~ms}$ and a spectral BW of $2 \mathrm{kHz}$, exciting the whole ${ }^{13} \mathrm{C}$-labelled nuclei window. The TR between each pulse excitation was set to $10 \mathrm{~s}$ and 26 averages per scan were acquired to ensure sufficient SNR. Seven scans were recorded; the first one at $t_{2}(1)$ of $1.69 \mathrm{~ms}$ and then increased in increments of $0.86 \mathrm{~ms}$ for the other scans. The spatial encoding was performed with a spiral out trajectory.

Images were acquired at two slice positions, 0 and $-4 \mathrm{~mm}$, leading to a transmit frequency $f_{T}$ of 0 and $-533 \mathrm{~Hz}$, respectively. For the shifted slice, acquisitions were recorded in the phasecontinuous mode, and for both, Acq-Switch and RF-Switch cases. The phase correction was only applied in the Acq-Switch case, following Eq. (4). For metabolic map reconstruction, the two-step process described in [15] was applied. Knowing the resonance frequencies of the metabolites, a k-space data was first reconstructed for each one fitting the signals acquired from all shots. These individual metabolite k-space data were then spatially reconstructed into metabolic maps.

Data processing for all experiments was performed on Matlab ${ }^{\circledR}$ R2019a (MathWorks, Natick, MA, USA) with home-made written scripts. The dataset of this work is available at the following link https://doi.org/10.15454/LY4VKC.

\section{Results}

Fig. 3 illustrates the application of both Acq- and RF- switch cases. It shows four columns, one for each metabolite. The ${ }^{13} \mathrm{C}$ maps are superimposed on a ${ }^{1} \mathrm{H}$ reference image of the phantom for clear syringe borders. The 1st row presents the control maps, i.e., generated at the centre of the magnet where $f_{T}=0$ (AcqSwitch case). The metabolic maps reconstructed for each of the metabolites fit with the corresponding syringe placement. As soon as the slice was shifted $\left(f_{T} \neq 0\right)$, it was impossible to reconstruct the correct metabolic maps (2nd row) without correcting the raw signal to cancel the effect of the spurious phase acquired due to the slice offset (3rd row). When using the RF-Switch case (4th row), such artefacts were not observed. The 0 -Switch case was unnecessary, hence not applied.

\section{Discussion}

Phase correction can be mandatory in some slice-selective IDEAL-type protocols depending on how the transmit/receive frequency switch is performed. Without phase correction, the slice selection or readout frequency offset can bias the CS-related phase evolution by adding different spurious phases during each shot.

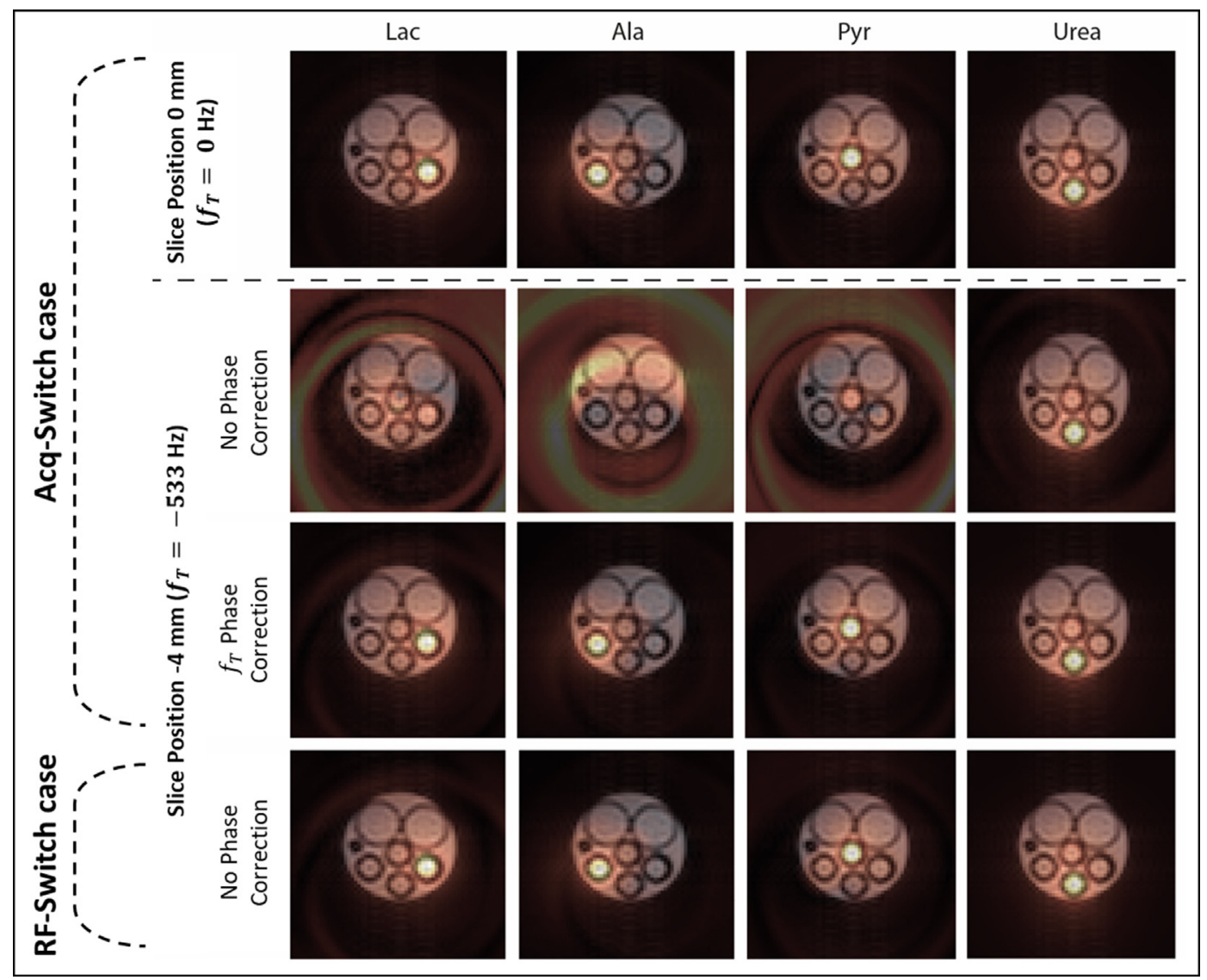

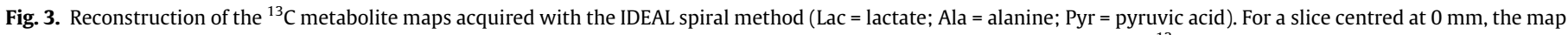

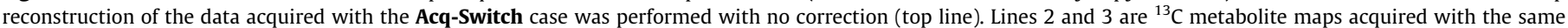

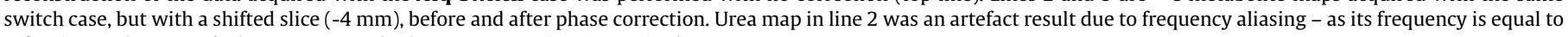
$3 f_{T}$. Line 4 , the RF-Switch case was used where no correction is required. 
In our practical case, only phase-continuous switch is allowed for the commutation from the transmit to the receive frequency $\left(f_{T} \rightarrow f_{R}\right)$ on the scanner. Although the proposed phase correction is valid to eliminate the possible spurious phases accumulated during the pulse sequence, it is desirable to avoid them. This is achieved by switching from the transmit frequency $f_{T}$ to the reference frequency at $t_{1}$. To do so, two options are possible: the RFSwitch case if and only if $f_{R}=0$, or the 0 -Switch case. In return, if the phase-coherent switch mode is at hand, one should favour the $f_{T} \rightarrow f_{R}$ commutation at $t_{2}$ before the acquisition, as it discharges one from any post-processing phase correction. Lastly, when the commutation mode is unknown, the only case requiring no correction is the proposed 0 -Switch case.

Generally speaking, these phase accumulation artefacts are encountered as soon as several images obtained from different excitations with different TEs are processed together to extract the CS-related information. For example, this can be the case in high-spatial resolution $\mathrm{B}_{0}$-mapping, e.g., for susceptibilityweighted imaging [26]. Indeed, to reach a sufficient Nyquist limit, the TE must be incremented by a small delay, thus requiring image acquisition from multiple excitations. In this case, the same issues may be encountered and the described solutions remain valid.

While the described approaches cancel unwanted phase accumulation due to frequency commutation's mode and position, they do not correct for other mechanisms leading to additional spurious phase. One of the critical sources of phase errors is any physiological motions. To obtain exploitable data, it is then important to avoid such movements during the signal encoding and acquisition. To do so, using a trigger on one of the physiological parameters, a navigator echo [27] or one of the solutions mentioned in the introduction [21-23] is still mandatory.

We focused on the phase effects in IDEAL-type pulse sequences, due to the transmit-receive commutation before the acquisition time. However, for methods using multiple RF pulses and being phase cycled, similar frequency issues may arise and lead to inoperative coherence selection, as they may spoil the desired phase cycle [24].

To conclude, we demonstrated that the position and the mode of the frequency commutation in the pulse sequence are critical to record only the CS-dependent phase evolution. This point is under-addressed in the scientific literature and therefore relatively unclear for MRSI users. Regardless of the hardware efficiency and the sequence implementation, we proposed several solutions to eliminate possible spurious phase in rapid metabolic imaging.

\section{Declaration of Competing Interest}

The authors declare that they have no known competing financial interests or personal relationships that could have appeared to influence the work reported in this paper.

\section{Acknowledgement}

This research has received funding from the Conseil Régional d'Auvergne, Grant No CPER 2015-2020-EPICURE. We thank Sascha Köhler and Jérôme Voiron for the useful discussions and for their mention of reference [24].

\section{References}

[1] M.A. Hadavand, D. Mayer, W.G. Chen, A. Wnorowski, M.M. Siddiqui, Role of metabolic imaging in diagnosis of primary, metastatic, and recurrent prostate cancer, Curr. Opin. Oncol. 32 (3) (2020) 223-231.

[2] M. Julia-Sape, A.P. Candiota, C. Arus, Cancer metabolism in a snapshot: MRS(I), NMR Biomed. 32 (10) (2019) 21.

[3] L. Mazuel, R.F. Schulte, A. Cladiere, et al., Intracerebral synthesis of glutamine from hyperpolarized glutamate, Magn. Reson. Med. 78 (4) (2017) 1296-1305.

[4] S. Gruber, E. Heckova, B. Strasser, et al., Mapping an extended neurochemical profile at 3 and 7T using accelerated high-resolution proton magnetic resonance spectroscopic imaging, Invest. Radiol. 52 (10) (2017) 631-639.

[5] R.F. Schulte, J.I. Sperl, E. Weidl, et al., Saturation-recovery metabolic-exchange rate imaging with hyperpolarized $\left[1-{ }^{13} \mathrm{C}\right]$ pyruvate using spectral-spatial excitation, Magn. Reson. Med. 69 (5) (2013) 1209-1216.

[6] J.J. Miller, A.Z. Lau, I. Teh, et al., Robust and high resolution hyperpolarized metabolic imaging of the rat heart at 7T with 3D spectral-spatial EPI, Magn. Reson. Med. 75 (4) (2016) 1515-1524.

[7] S.B. Reeder, J.H. Brittain, T.M. Grist, Y.F. Yen, Least-squares chemical shift separation for ${ }^{13} \mathrm{C}$ metabolic imaging, J. Magn. Reson. Imaging 26 (4) (2007) 1145-1152.

[8] T.A. Bley, O. Wieben, C.J. Francois, J.H. Brittain, S.B. Reeder, Fat and water magnetic resonance imaging, J. Magn. Reson. Imaging 31 (1) (2010) 4-18.

[9] S.B. Reeder, I. Cruite, G. Hamilton, C.B. Sirlin, Quantitative assessment of liver fat with magnetic resonance imaging and spectroscopy, J. Magn. Reson. Imaging 34 (4) (2011) 729-749.

[10] H.H. Hu, H.E. Kan, Quantitative proton MR techniques for measuring fat, NMR Biomed. 26 (12) (2013) 1609-1629.

[11] K. Kuroda, Non-invasive MR thermography using the water proton chemical shift, Int. J. Hyperthermia 21 (6) (2005) 547-560.

[12] N. McDannold, K. Hynynen, K. Oshio, R.V. Mulkern, Temperature monitoring with line scan echo planar spectroscopic imaging, Med. Phys. 28 (3) (2001) 346-355.

[13] T.R. Brown, B.M. Kincaid, K. Ugurbil, NMR chemical-shift imaging in 3 dimensions, Proc. Natl. Acad. Sci. U.S.A.-Biol. Sci. 79 (11) (1982) 3523-3526.

[14] P. Mansfield, Spatial mapping of the chemical shift in NMR, Magn. Reson. Med. 1 (3) (1984) 370-386.

[15] F. Wiesinger, E. Weidl, M.I. Menzel, et al., IDEAL spiral CSI for dynamic metabolic MR imaging of hyperpolarized $\left[1-{ }^{13} \mathrm{C}\right]$ Pyruvate, Magn. Reson. Med. 68 (1) (2012) 8-16.

[16] W.W. Jiang, M. Lustig, P.E.Z. Larson, Concentric rings K-space trajectory for hyperpolarized C-13 MR spectroscopic imaging, Magn. Reson. Med. 75 (1) (2016) 19-31.

[17] S.B. Reeder, A.R. Pineda, Z.F. Wen, et al., Iterative decomposition of water and fat with echo asymmetry and least-squares estimation (IDEAL): application with fast spin-echo imaging, Magn. Reson. Med. 54 (3) (2005) 636-644.

[18] S.B. Reeder, Z.F. Wen, H.Z. Yu, et al., Multicoil Dixon chemical species separation with an iterative least-squares estimation method, Magn. Reson. Med. 51 (1) (2004) 35-45.

[19] J.Z. Wang, A.J. Wright, D.E. Hu, R. Hesketh, K.M. Brindle, Single shot threedimensional pulse sequence for hyperpolarized ${ }^{13} \mathrm{C}$ MRI, Magn. Reson. Med. 77 (2) (2017) 740-752.

[20] P.E.Z. Larson, A.B. Kerr, A.P. Chen, et al., Multiband excitation pulses for hyperpolarized ${ }^{13} \mathrm{C}$ dynamic chemical-shift imaging, J. Magn. Reson. 194 (1) (2008) 121-127.

[21] P.E.Z. Larson, S. Hu, M. Lustig, et al., Fast dynamic 3D MR spectroscopic imaging with compressed sensing and multiband excitation pulses for hyperpolarized ${ }^{13} \mathrm{C}$ studies, Magn. Reson. Med. 65 (3) (2011) 610-619.

[22] S. Posse, G. Tedeschi, R. Risinger, R. Ogg, D. Lebihan, High speed ${ }^{1} \mathrm{H}$ spectroscopic imaging in human brain by echo planar spatial-spectral encoding, Magn. Reson. Med. 33 (1) (1995) 34-40.

[23] L. Cordero-Grande, E.J. Hughes, J. Hutter, A.N. Price, J.V. Hajnal, Threedimensional motion corrected sensitivity encoding reconstruction for multishot multi-slice MRI: application to neonatal brain imaging, Magn. Reson. Med. 79 (3) (2018) 1365-1376.

[24] F. Hennel, The effective phase of soft RF pulses, Concepts Magn. Reson. Part A 43 (4) (2014) 127-137.

[25] W.A. Edelstein, J.M.S. Hutchison, G. Johnson, T. Redpath, Spin warp NMR imaging and applications to human whole-body imaging, Phys. Med. Biol. 25 (4) (1980) 751-756

[26] S.J. Wang, D.J. Peterson, J.C. Gatenby, W.B. Li, T.J. Grabowski, T.M. Madhyastha, Evaluation of field map and nonlinear registration methods for correction of susceptibility artifacts in diffusion MRI, Front. Neuroinform. 11 (2017) 9.

[27] R.J. Ordidge, J.A. Helpern, Z. Qing, R.A. Knight, V. Nagesh, Correction of motional artifacts in diffusion-weighted MR images using navigator echoes, Magn. Reson. Imag. 12 (3) (1994) 455-460. 